\title{
An inventory location routing model with environmental considerations
}

\author{
Sanaa Lerhlaly $^{1,2, *}$, Maria Lebbar $^{3}$, Hamid Allaoui ${ }^{1}$, Sohaib Afifi ${ }^{1}$, and Driss Ouazar $^{2,3}$ \\ ${ }^{1}$ LGI2A, Université d'Artois, France \\ ${ }^{2}$ LASH, Ecole Mohammadia d'ingénieurs Université Mohammed V de Rabat .Morocco \\ ${ }^{3}$ SYSCOM, Ecole Nationale Supérieure des Mines de Rabat, Morocco
}

\begin{abstract}
In this work we consider an integrated Inventory Location Routing problem ILRP. The objective is to select depots to open, the routes to perform distribution from the capacitated depots to the clients by a homogeneous fleet of vehicles and over a multi-period planning horizon. To solve the problem, an exact model is designed in order to minimize the total cost (location, routing and inventory) and the routing $\mathrm{CO} 2$ emissions, while respecting transport and storage constraints for a hazardous material. We have also studied an industrial real case to illustrate the problem.
\end{abstract}

\section{Introduction and literature review}

The design of efficient supply chains has become more challenging in view of environmental regulations, especially for Hazardous materials (Hazmat) supply chains. The decisions for supply chain management can be categorized into three types: strategic, tactical, and operational. The classical approach for the optimization of logistics systems is the decomposition of the system into sub problems according to these decision levels. Facility location problems (strategic level) deal with defining the number, the location and the capacity of the warehouses, in order to meet customer demand at lower cost. This problem was reviewed by [1-3]. The vehicle routing problem (VRP) (tactical or operational level) was studied in [4-7]. The solving techniques used for this problem were summarized by [8-10].

This approach has many advantages such as simplification of modeling and problem solving techniques. This can, though, lead to a sub optimality of solutions, because it doesn't take account of the entire problem [11].

In order to a global view of the supply chain design, some papers integrate the three components: location, routing and inventory. The problem becomes more complex, but it is important to anticipate interactions between decisions levels.

The Inventory Location-Routing Problem (ILRP) can be considered as an approach for integrating the three components (depot location, vehicle routing and inventory management) into one model, in order to optimize the supply chain design and minimize the total costs. In $[12,13]$, authors presented a location-routing problems (LRP) including nonlinear inventory costs in the objective function. They consider a single product and multi-depot with unlimited capacity and stochastic demand. In order To solve this problem, Lui and Lee [12] used a two-phase heuristic method, while Liu and Lin [13] proposed a hybrid heuristic based on the Tabu Search (TS) and the Simulated Annealing (SA). Another nonlinear stochastic model was studied by Shen and Qi [14] in order to integrate the location, inventory, and the routing approximate costs. Javid and Azad [15] presented a stochastic model in order to find simultaneously the decisions of location, allocation, inventory at depots and routing. The authors proposed an exact solution and then a heuristic method based on hybridization of Tabu Search (TS) and Simulated Annealing (SA). Regarding the deterministic demand, Ambrosino and Scutella [16] used a linear programming relaxation for the ILRP. Hiassat and Diabat [17] studied an inventory location routing problem with deterministic demand for perishable product. They proposed a multiperiod model formulated as Mixed Integer Program (MIP). Recently, Guerrero et al. [18] presented an algorithm for a multi-period ILRP with deterministic demand. They proposed a hybrid approach in order to optimize simultaneously location of depots, allocation of retailers to depots, inventory at both depots and retailers, and multi-period routing. The algorithm optimizes globally the components of the problem by sharing information between decisions levels. Guerrero et al. [19] investigated a Relax and Price heuristic for the same problem. They coordinated the generation of new columns in the pricing problem and updated Lagrangian multipliers. Results show important cost savings and efficient computation if compared to the previous heuristics. Ying Zhang et al. [20] proposed a hybrid metaheuristic solution in order to solve an ILRP. They considered a multiple depots and geographically dispersed customers. Nekooghadirli et al. [21] studied a bi-objective location-routing-inventory (LRI) model

\footnotetext{
* Corresponding author: sanaalerhlaly@gmail.com
} 
considering multi-period, stochastic demands, multiproduct and probabilistic travelling time among customers. Authors presented a mixed integer nonlinear programming model in order to minimize the total cost and maximize mean time for delivering commodities to customers.

This paper deals with an integrated Inventory Location Routing Problem (ILRP) considering a deterministic demand and a multi-period horizon of decisions. In order To take into account risks related to the use of Hazmat, we consider storage constraints at depots and clients, small capacity vehicles, and routes with fixed number of customers which must not be exceeded.

\section{Problem definition}

We are studying a supply chain design problem for Hazmat. We have to distribute a hazardous product from a single manufacturer to a set of customers, through a set of intermediate depots with homogeneous fleet. This aims to minimize both of the total cost and the routing $\mathrm{CO} 2$ emissions. The total costs signify the depots opening, the routing, and the clients inventory costs.

A first model for this problem was presented in S.Lerhlaly et al. [22]. The problem is formulated as a set covering model whose the decision variables are the routes. A case study is also provided.

Let: $(I)$ denote the set of $(m)$ candidate depots. $(J)$ denote the set of $(n)$ customers, $(T)$ denote the set of $(p)$ periods in the planning horizon, and $(K)$ denote the set of vehicles. $V=\left\{J_{U} I\right\}$ is the set of nodes in the graph $G=$ $(V ; A ; C) . C$ is the cost matrix $\left(c_{i j}\right)$ associated to the traveling cost from node $\mathrm{i}$ to node $\mathrm{j}$ in the set of $\operatorname{arcs} A$.

The hazardous materials regulations limit the storage and the transportation quantities which increase the transportation frequency, and augment the environmental impacts due to $\mathrm{CO} 2$ emissions. That is why it is important to add these environmental impacts to the objective function. The estimation of $\mathrm{CO} 2$ emission for transportation requires complex calculations, because of the difficulty of quantifying some variables such as driving style, weather conditions, congestion...[23,24]. In order To calculate the emissions factor $\mathrm{CO} 2$, We are using a method similar to that used by S. Ubeda et al. [25].

\subsection{Parameters}

Oi Opened cost of depot $i \in I$

$f \mathrm{jt}$ Inventory holding cost of customer $j \in J$ in time period $t \in T$

$F$ vehicles cost

Cap Capacity of vehicule $K$

$d \mathrm{jt}$ Demand of customer $j \in J$ in time period $t \in T$

$S_{\mathrm{j} 0}$ Inventory level at customer $j \in J$ at the beginning of time period $t=1$

$b_{\mathrm{j}}$ Maximum inventory level at customer $\mathrm{j}$

$C p d_{\mathrm{i}}$ Maximum storage capacity at depot $\mathrm{i}$

MaxC Maximum number of customer by route

$E \mathrm{j} \mathrm{CO} 2$ emissions factor $\alpha, \beta, \lambda, \Omega$ Weights factor associated with opening cost, transportation cost, $\mathrm{CO} 2$ emission ,inventory cost.

\subsection{Decisions variables}

$y_{\mathrm{i}}=1$ if depot $\mathrm{i} \in \mathrm{I}$ is opened and 0 otherwise

$x_{\mathrm{ijkt}}=1$ if the arc $(\mathrm{i}, \mathrm{j})$ used in the route performed by the vehicle $\mathrm{k} \in \mathrm{K}$ on period $\mathrm{t} \in \mathrm{T}$ and 0 otherwise

$f_{\mathrm{ij}}=1$ if clients $\mathrm{j}$ is assigned to depot $\mathrm{I}$ and 0 otherwise

$S_{\mathrm{jt}}=$ Inventory holding of customer $\mathrm{j} \in \mathrm{J}$ in the end time period $\mathrm{t} \in \mathrm{T}$

$Q_{\mathrm{ijkt}}=$ Quantity delivered from depot $\mathrm{i} \in \mathrm{I}$ to customer $\mathrm{j} \in$ $\mathrm{J}$ by vehicle $\mathrm{k} \in \mathrm{K}$ in time period $\mathrm{t} \in \mathrm{T}$.

\subsection{Model}

$$
\begin{gathered}
\min \alpha \sum_{i \in I} o_{i} y_{i} \\
+\beta \sum_{i \in V} \sum_{j \in V} \sum_{k \in K} \sum_{t \in T} c_{i j} x_{i j k t}+\sum_{i \in I} \sum_{j \in J} \sum_{k \in K} \sum_{t \in T} F x_{i j k t} \\
+\lambda \sum_{i \in V} \sum_{j \in V} \sum_{k \in K} \sum_{t \in T} c_{i j} e_{j} x_{i j k t}+\Omega \sum_{j \in J} \sum_{t \in T} m_{j t} S_{j t}
\end{gathered}
$$

Subject to

$$
\begin{aligned}
& \sum_{i \in I} \sum_{j \in J} Q_{i j k t} \leq \operatorname{Cap} \quad \forall k \in K, \forall t \in T \\
& \sum_{j \in V} x_{i j k t}-\sum_{j \in V} x_{j i k t}=0 \quad \forall k \epsilon K, \quad \forall t \in T, \forall i \epsilon V \\
& \sum_{i \in j} \sum_{k \in K} x_{i j k t} \leq 1 \quad \forall j \epsilon J, \quad \forall t \in T \\
& \sum_{i \in j} \sum_{k \in K} x_{j i k t} \leq 1 \quad \forall j \epsilon J, \quad \forall t \epsilon T \\
& \sum_{i \in I} \sum_{j \in J} x_{i j k t} \leq 1 \quad \forall k \in K, \quad \forall t \in T \\
& \sum_{i \in I} f_{i j}=1 \quad \forall j \epsilon J \\
& \sum_{a \in J} x_{i a k t}+\sum_{a \in V} x_{a j k t} \leq 1+f_{i j} \\
& \forall j \in J, \forall i \in I, \forall k \in K, \forall t \in T \\
& \sum_{j \in J} \sum_{k \in K} Q_{i j k t} \leq c p d_{i} * y_{i} \quad \forall i \epsilon I, \quad \forall t \in T \\
& S_{j t-1}+\sum_{i \in I} \sum_{k \in K} Q_{i j k t}=d_{j t}+S_{j t} \quad \forall j \epsilon J, \quad \forall t \epsilon T \\
& S_{j t} \leq b_{j} \quad \forall j \epsilon J, \quad \forall t \in T
\end{aligned}
$$




$$
\begin{gathered}
\sum_{i \in V} \sum_{j \in V} x_{i j k t}-1 \leq \operatorname{Max} C \quad \forall t \in T, \quad \forall k \in K \\
\sum_{j \in S} \sum_{j \in S} x_{i j k t} \leq|S|-1 \quad \forall S \epsilon J, \forall k \epsilon K, \forall t \\
\sum_{t \in T} \sum_{k \in K} Q_{i j k t} \leq M * f_{i j} \quad \forall i \epsilon I, \quad \forall j \epsilon J \\
Q_{i j k t} \leq M * \sum_{u \in J} x_{i u k t} \quad \forall i \in I, \forall j \in J, \forall t \in T, \forall k \in K
\end{gathered}
$$

Objective function (Equation1) includes the sum of location, transportation, inventory costs and $\mathrm{CO} 2$ emissions. The first term represents the opening depots fixed cost, the transportation costs are minimized by second and third terms, $\mathrm{CO} 2$ emissions costs is minimized by the fifth term and the last one represents the inventory holding cost. Constraint (2) indicates the capacity constraints of vehicles. Constraints (3-5) present vehicle flow conservation. Constraint (6) forces each vehicle to perform one route per period at the most. Constraint (7) guarantees that each customer must be allocated to a single opened depot. Constraint (8) state that a retailer $j$ can't be linked to a depot $i$ if $j$ is not assigned to depot $i$. The capacity of each depot is presented in Equation (9).Equation (10) represents the inventory balance. Equation (11) ensures that the inventory level at a customer never exceeds maximum storage limits due to the Hazmat regulations. Equation (12) respects the maximum number of customers per route. The traditional subtour elimination constraints are presented in Equation (13). Constraints (14) (15) guarantee that if a retailer is served on period $t$ with route $\mathrm{k}$, it must be visited.

To validate the proposed model, a real case study has been developed in order to optimize the design of chlorine supply chain (for a leading company in the drinking water distribution sector in Morocco). This company use chlorine for water disinfection. The problem considers 10 delivery points (Clients).The maximum number of clients per route is 3 . The Number of candidate depots is 5 . The planning horizon is three time periods.

Table 1. Simulation results

\begin{tabular}{|c|c|c|c|c|c|c|}
\hline $\begin{array}{c}\text { Eco2 } \\
\text { weight }\end{array}$ & Total cost & D.C & N.D & R.C & I.C & Eco2 \\
\hline$\lambda=0$ & 718498,6 & 700000 & 1 & 7458 & 1040,6 & 3898,7 \\
\hline$\lambda=100$ & 719287,1 & 700000 & 1 & 7334 & 1953,1 & 3586,4 \\
\hline$\lambda=1000$ & 2156245,6 & 2140000 & 3 & 4537 & 1708,6 & 2862,2 \\
\hline$\lambda=10000$ & 2936148,1 & 2920000 & 4 & 4438 & 1710,1 & 2026,7 \\
\hline
\end{tabular}

The results of simulations are reported in Table1. The first column of this table indicates the weight of the $\mathrm{CO} 2$ emissions. The second column presents the total cost. Columns: D.C, N.D, R.C, I.C respectively indicate the costs and number of opened depots, the routing and inventory costs. The last column gives the $\mathrm{CO} 2$ emissions quantity.

The results show that the total cost and the number of selected depots increased as the weight of the transportation $\mathrm{CO} 2$ emissions increased (Fig.1, Fig.2).

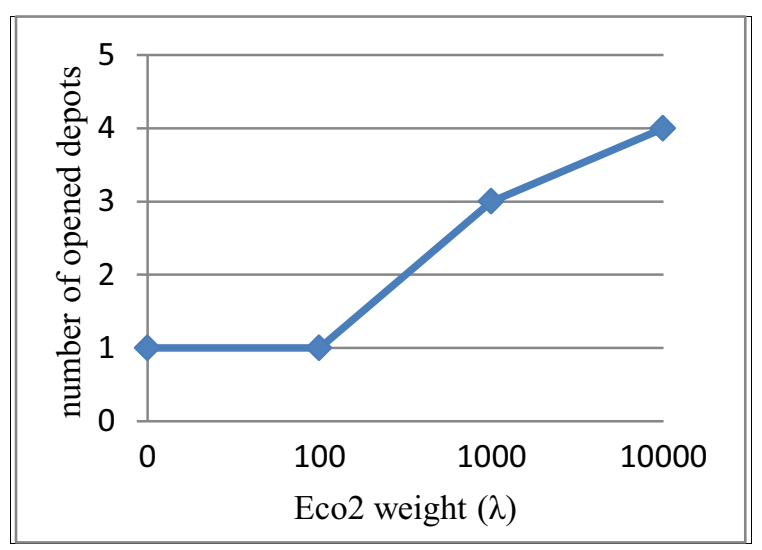

Fig. 1. Number of opened depots vs $\mathrm{Co} 2$ emissions weight

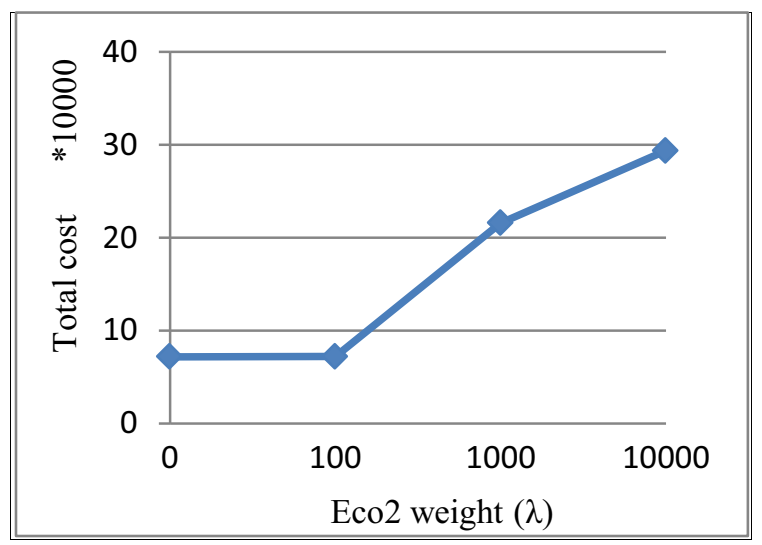

Fig. 2. Total cost vs $\mathrm{Co} 2$ emissions weight.

\section{Conclusion}

We present an inventory location routing problem (ILRP) that optimizes the operating costs, and routing's $\mathrm{CO} 2$ emissions taking into account Hazmat constraints. We modeled the problem as an exact integer linear program. The developed model is applied to a case study of Hazmat supply chain to demonstrate its applicability. Preliminary results are promising for medium size instances. The future work is both the development of a heuristic method, based on a guided generation of routes, and also the testing of some larger scale instances.

\section{References}

1. Z. Drezner, Springer, New York, (1995).

2. S.H. Owen, M. S. Daskin, European Journal of Operational Research, (1998), 111(3), 423-447.

3. M.T. Melo, S.Nickel, F. Saldanha-da-Gama, European Journal of Operational Research, (2009), 196(2), 401-412. 
4. Bodin, L.D., Golden, B.L., Assad, A.A., and Ball, M.O. Computers and Operations Research (1983), 10, 69-211.

5. G. Laporte, H.Osman, Annals of Operations Research (1995), 61, 227-262.

6. G. Laporte. Transportation Science, (2009), 43(4), 408-416.

7. M. Drexl, European Journal of Operational Research, (2013), 227(2), 275 -284.

8. G. Laporte, (European Journal of Operational Research 1992), 59, 345-358.

9. G. Laporte, M. Gendreau, J. Potvin, F.Semet, International transactions in operational research, (2000), 7(4-5), 285-300.

10. P. Toth, D. Vigo, Discrete Applied Mathematics, (2002), 123(1-3), 487-512.

11. C. Prodhon, PhD Thesis, University of Technology of Troyes, France, (2006)

12. S.C. Liu, S.B. Lee, The International Journal of Advanced Manufacturing Technology, 22 (2003), 941-950.

13. S. Liu, C. Lin, The International Journal of Advanced Manufacturing Technology, 26 (2005), 372-381.

14. Z.J.M. Shen, L. Qi, European Journal of Operational Research, 179 (2007), no. 2, 372-389.

15. A. Ahmadi Javid, N. Azad, Transportation Research Part E: Logistics and Transportation Review, 46 (2010), no. 5, 582 - 597.

16. D. Ambrosino, M.G. Scutell a, European Journal of Operational Research 165 (2005), no. 3, 610-624.

17. A. Hiassat, A. Diabat, Proceedings of the 41st International Conference on Computers and Industrial Engineering, (2011).

18. W.J. Guerrero, C. Prodhon, N. Velasco, C.A. Amaya, International Journal of Production Economics, 146 (2013), no. 1, 359-370.

19. W.J. Guerrero, N. Velasco, C. Prodhon, C.A. International Transactions in Operations Research, 22 (2015), 129-148.

20. Zhang et al., 2014, Y. Zhang, M. Qi, L. Miao, E. Liu, Transport. Res. Part E: Logist. Transport. Rev., 70 (2014), pp. 305-323

21. Nekooghadirli, N., Tavakkoli-Moghaddam, R., Ghezavati, V., Javanmard, S. Comput. Indust. Eng (2014)., 76, 204-221.

22. Sanaa Lerhlaly, Maria Lebbar, Hamid Allaoui, Driss Ouazar, Sohaib afifi, Contemporary Engineering Sciences, Vol. 9,(2016), no.7,303-314.

23. A. Palmer, Logistics Research Network Conference Proceedings, University of Hull, Hull, (2007), 2732.

24. T.V. Van Woensel, R. Creten, N. Vandaele, Production and Operations Management, 10 (2001), 207-223.

25. S. Ubeda, F.J. Arcelus, and J. Faulin, International Journal of Production Economics, 131 (2011), 4451. 